\title{
The Features of the Reference Frame Concomitant to the Cosmic Microwave Background
}

\author{
V. M. Svishch
}

\section{ABSTRACT}

The features of reference frame, concomitant to the cosmic microwave background, immobile relatively cosmic microwave background, are considered. It is shown that the features of reference frame, concomitant to the cosmic microwave background (CMB), are determined by its properties. Any other object in the Universe and reference frame concomitant to it, is immersed in the $\mathrm{CMB}$ and moves relative to the reference frame concomitant to microwave background radiation. The zero pecular velocity of the reference frame concomitant to the microwave background radiation is analogous to the zero temperature on the Kelvin scale. Time in it is most rapid in relation to the time in any other reference frame, observable and measurable in any of them. The features of time, pecular speed, relative speed of two inertial RF, stellar aberration, and Doppler effect in the reference frame concomitant to the microwave background radiation are considered. According to the determined relative velocity of the two reference systems and the peculiar velocity of the reference system with the observer, the components of their relative velocity are determined. Determining the components of the relative velocity of the reference frames with determining the synchronous time for all points at any time in the reference frame concomitant to microwave background radiation, allows us to investigate the possibility of determining the speed of light "one way" and using it to navigate vehicles in distant space. Stability of angular location of heterogeneities of $\mathrm{CMB}$ in reference frame concomitant to CMB, allows us to use these heterogeneities for the increase of exactness of astronomic reference frames HCRF and ICRF.

Keywords: cosmic microwave background (CMB), reference frame (RF), reference frame concomitant to the microwave background radiation (CMBRF), preferential reference frame, pecular velocity, light aberration, Doppler effect.

Published Online: November 04, 2021

ISSN: $2684-4451$

DOI : 10.24018/ejphysics.2021.3.6.115

V. M. Svishch*

Kharkov National University of Radio Electronics, Ukraine.

(e-mail: vladimir.svishh@ rambler.ru)

*Corresponding Author

\section{INTRODUCTION}

The representation of a reference frame (RF) is closely related to the notion of motion. Therefore, any reference frame consists of an object it accompanies, a system of coordinates, and a clock. A reference frame containing its own clock is considered complete. For any reference frame, a connection with an object is specified, i.e., it (bound) accompanies the object.

From several reference frames concomitant to the same object, depending on the problems to be solved, a reference frame preferable for these problems (inertial - non-inertial, Lagrangian-Eulerian, astronomical reference frames, technical) is selected, taking into account the properties of the object, the method of research.

Preferable reference frames concomitant to various objects, convenient for their description and related processes (planes, rockets, satellites, Earth, Sun) are constantly developing and widely used.

The concept of motion relativity appeared with the development of mechanics thanks to Galileo. The medium of absolute reference of motion was still considered a hypothetical ether. Time was considered invariable, unified.

Fizeau's experiments (1851) fully confirmed the partial entrainment of the ether with the light flux, according to the notions of the time [1].

The experiments of Michelson (1881), Michelson-Morley (1887) to determine the movement of the Earth relative to the ether were the culmination of the search for motion relative to the ether [2], [3]. Similar experiments, with increasing their accuracy, continue to the present time [4], [5].

The works of Lorentz, Poincaré, Einstein and the results of the Michelson-Morley experiments made it possible to abandon the ether completely [6]-[8].

A. Einstein, having investigated the concept of time and simultaneity and having realized the universality of the connection between space and time, created the general theory of relativity. This allowed him to describe the universe as a physical object by the equations of General Relativity Theory [9], [10].

The solutions of these equations by Friedman (1922) 
pointed to the possibility of expansion of the Universe in space and time, its non-stationarity.

Studying the model of the Universe expansion after the Big Bang, Gamov calculated the temperature of the cosmic microwave background in our time $-3^{\circ} \mathrm{K}$ [11].

Penzias and Robert Wilson discovered and accepted the $\mathrm{CMB}$, confirming its existence. As a result, the CMB produced a "record" of the expansion process from the Big Bang to the present day.

Observations of extragalactic objects made it possible to associate with them the coordinate axes of two barycentric reference frames: ICRF - in the radio range and HCRF - in the visible range [12].

The CMB measurements from the RELIKT-1 (USSR, 1983-1984), COBE (USA, 1989-1996), WMAP (USA, 20012009), Planck (EU, 2009-2010) satellites became a treasure trove of information about the Universe [13]-[16]. The CMB directional temperature distribution has helped to determine the magnitude and direction of the Earth's peculiar velocity, taking into account even the velocity of its rotation around the Sun [17]-[19].

As a result, taking into account Hubble's law of universe expansion, we had to switch from the Big Bang model to the Standard Cosmological Model (ACDM).

With the discovery, CMB began to be regarded as a contender for the role of an object for the accompanying, dedicated reference frame. It immediately began to claim the role of a universal dedicated reference frame, the "new ether" as early as 1975 [20].

Thus, the rapid development of the Standard Cosmological Model, confirmed by the Hubble Law and CMB discoveries, made it possible to create the reference frames ICRF, HCRF associated with extragalactic objects and to consider the possibilities of the reference frame concomitant to CMB.

Let us consider the features of the reference frame concomitant to the microwave background radiation.

\section{The ReFERENCE Frame CONCOMITANT To CMB}

By definition, the reference frame concomitant to the microwave background radiation is connected with the object - the microwave background radiation and is stationary with respect to the microwave background radiation.

Properties of the microwave background radiation and peculiarities of its relations with space-time determine peculiarities of the reference frame concomitant to CMB.

After recombination of electrons and protons in the process of separation of radiation from plasma, the Universe reached the order of magnitude of the currently observed Universe. Thus, microwave background radiation appeared "everywhere" [20]-[22].

The defining property of microwave background radiation is its isotropy. At any point of space, the CMB photons arriving from different directions have a temperature that does not differ from the average at the level of 10\-4.

From any fixed direction the spectrum of the $\mathrm{CMB}$ photons has a Planckian distribution [22].

In addition, the $\mathrm{CMB}$ power spectrum has a large information capacity [17], [18], [22].

First of all, the single post-recombination time at each observation point, which has elapsed from the moment when photons leave thermal equilibrium in the plasma until the moment when they are observed, is determined by the CMB temperature.

The dipole component of the CMB frequency shift determines the magnitude and direction of the observer's velocity relative to $\mathrm{CMB}$, the observer's pecular velocity.

The microwave background radiation, representing the medium with such properties, fills the whole Universe with all its objects

These basic parameters of CMB and determine the properties of the accompanying reference frame.

This is the only isolated, at the given stage of cognition, the inertial system with the zero pecular velocity, the dipole component of an observer resting in it is equal to zero in any direction. It covers all other objects of the Universe and their related reference frames. Any other object of the Universe and its accompanying reference frame are immersed in $\mathrm{CMB}$ and move relative to the reference frame concomitant to CMB.

Besides, if all accompanying reference frames, except for the cosmological reference frame concomitant to Hubble flux, go beyond the accompanying object, then the reference frame concomitant to CMB is all immersed in CMB.

Or in other words, all reference frame concomitant to $\mathrm{CMB}$, is filled with isotropic microwave background radiation with the above basic parameters at any point of it.

Any object of the Universe is immersed in isotropic microwave background radiation with an energy density different from zero.

Thus, the reference frame concomitant to any Universe object, is within the reference frame concomitant to the microwave background radiation.

The properties of the electromagnetic microwave background radiation are well enough studied and available for measurement in each point of it. This defines the features of the reference frame concomitant to the microwave background radiation, gives it, to a certain extent, universality and distinguishes it from all other reference frames.

The reference frame concomitant to microwave background radiation, is not a preferential reference frame, but a dedicated one. While there are many preferential reference frames depending on the tasks to be solved, a dedicated reference frame is only one of its kind.

Let us consider in detail at what makes this reference frame stand out.

\section{FEATURES OF THE REFERENCE FRAME CONCOMITANT TO THE MICROWAVE BACKGROUND RADIATION}

Since the reference frame concomitant to CMB is all filled with isotropic microwave background radiation with the above basic parameters at any point of it, all reference frames concomitant to the any Universe object are within it. It is the only, at this stage of cognition, reference frame that covers (encompasses) all other reference frames.

\section{A. Velocity}

The reference frame concomitant to the microwave background radiation, is inertial at any point. The dipole component of coordinates and its Hubble expansion in this reference frame are absent (equal to zero). It has no pecular 
velocity, if its coordinates concomitant to Hubble expansion.

Thus, reference frame concomitant to microwave background radiation, is an inertial system with the zero pecular velocity. All other reference frames move in the reference frame concomitant to the microwave background radiation with a pecular velocities $v_{p i}$ relative to CMB.

Therefore, it is natural to consider the reference frame concomitant to CMB as stationary $v_{0 n}=0$.

Any other Universe object and its accompanying reference frame, move relative to $\mathrm{CMB}$ with a pecular velocity causing a dipole component of $\mathrm{CMB}$ in it. Its velocity is limited on one side by the zero pecular velocity and on the other side by the velocity of light $0 \geq v_{p i} \leq c$.

\section{B. Time}

The reference frame concomitant to $\mathrm{CMB}$, is distinguished by its time. Time in this reference frame is determinable at any moment at any point by the CMB temperature due to its isotropy. It is provided first of all by the Planckian distribution of the spectrum of the CMB photons coming from any fixed direction. An observer in any reference frame can determine at any moment the time in the reference frame concomitant to the microwave background radiation, based on an analysis of the CMB photon spectrum at that moment. There is no such possibility in any other reference frame.

At any point of the reference frame concomitant to CMB, the time is the same at the observed moment (synchronously for all points). This makes it possible to synchronize the clocks of any other $\left(v_{p i}>0\right)$ reference frames. It determines the time elapsed since the recombination of the primary plasma after inflation, in accordance with the standard cosmological model (ACDM).

According to the theory of relativity, time $t_{0 n}$ in it is fastest relative to time $t_{p i}$ in any other reference frame, having $v_{p i}>0$.

Denoting the time interval in reference frame concomitant to the microwave background radiation, as $\Delta t_{0 n}$, and the same time interval in any other reference frame, as $\Delta t_{p i}$ we get

$$
\Delta t_{0 n}=\Delta t_{p i} \sqrt{1-\frac{v_{p i}^{2}}{c^{2}}}
$$

The cosmological reference frame concomitant to Hubble Flux, is close to the reference frame concomitant to the microwave background radiation. It is also filled with the substance, to which concomitant and it contains all objects of the Universe. Since CMB traces the expansion of space since the radiation detachment, the coordinate axes of the reference frame concomitant to $\mathrm{CMB}$ trace the expansion of space in accordance with Hubble's law. Thus, they coincide in coordinates. However, the properties of the substance of the concomitant to cosmological reference frame are little known at present. This does not allow us to talk about its other properties.

That is, the cosmological reference frame is preferential for a certain range of problems in cosmology [22].

On the other hand, the astronomical reference frames (barycentric reference coordinate systems ICRF - in the radio range and HCRF - in the visible range) are close to the reference frame concomitant to CMB. These reference frames use distant extragalactic objects with cosmological redshift $z \leq 10$. The great distance from objects provides their small angular departures caused by the tangential components of their peculiar velocities. The CMB cosmological redshift $(z \geq 1000)$ provides stability of the angular location of its inhomogeneities caused by density fluctuations at the radiation detachment stage as well as at the inflation stage. This makes it possible to use them to improve the accuracy of reference frames ICRF and HCRF (e.g., over time to correct the angular position of reference objects with respect to the nearest $\mathrm{CMB}$ inhomogeneities, or to use the inhomogeneities themselves as reference objects).

The reference frame concomitant to $\mathrm{CMB}$ considers (reflects) only the pecular velocities of the Universe objects. Since it accompanies the isotropic homogeneous CMB, its coordinate axes remain oriented in the radial direction along the CMB. For the reference frame concomitant to CMB, at any transfer of the origin, or any rotation of axes, the pecular velocities of the Universe objects and their distribution remain invariant. The reference frame concomitant to $\mathrm{CMB}$ is the only one, which reflects only the pecular velocities of the Universe objects without Hubble components of velocities and satisfies the "cosmological principle".

The reference frame concomitant to $\mathrm{CMB}$, due to the above features, first of all speed and time, is complete, with its own clocks, and stands out from all reference frames.

Thus, we have a physical reference frame, in which all the Universe objects and their accompanying reference frames move at pecular velocities without including the Hubble velocity projections. Their velocity is limited on one side by the zero pecular velocity, and on the other side by the speed of light.

In a sense, the velocity of the reference frame concomitant to $\mathrm{CMB}$ and the velocities of all other reference frames are analogous to the Kelvin temperature scale, where the zero pecular velocity of the reference frame concomitant to $\mathrm{CMB}$, corresponds to $0^{0} \mathrm{~K}$. However, the velocity is a vector quantity and cannot exceed the speed of light $v_{p} \leq c$.

\section{Relative Velocity}

Let us consider the effect of the possibility of determining the pecular velocity of the observer in the analysis of relative motion.

Let the observer be in the inertial reference frame 1 , the pecular velocity $v_{p 1}$ of which is known. It is determined by the dipole component of the CMB. Using the Doppler effect, the velocity $v_{1,2}$ of reference frame 1 with respect to another inertial reference frame 2 , the pecular velocity of which is unknown, is also determinable. The relative velocity of reference frames 1,2 $v_{1,2}$ is equal to the difference of projections of its pecular velocities 1,2 to the direction of the relative velocity of reference frame 1 taking into account their directionality $v_{p 1} \cos \alpha_{1}-v_{p 2} \cos \alpha_{2}=v_{1,2}$. The velocity of reference frame $1 v_{p 12}$ to reference frame 2 will be equal to the projection of the pecular velocity of reference frame 1 
$v_{p 12}=v_{p 1} \cos \alpha_{1}$, then the velocity of reference frame 2 to

reference frame $1 v_{p 21}$ will be equal to $v_{p 21}=v_{p 2} \cos \alpha_{2}$, and $v_{p 21}=v_{p 1} \cos \alpha_{1}-v_{12}$.

Thus, the components $v_{p 12}, v_{p 21}$ of the relative velocity due to the motion of reference frame 1 and reference frame 2 can be determined by the observer in reference frame 1 . Determining the reference frame 1 velocity using the Doppler effect does not make it possible to determine these components of its velocity.

The determination of the components of the inertial reference frames relative velocity, with the determination of the synchronous time at any time in any point of the reference frame concomitant to $\mathrm{CMB}$ allows us to consider the possibility of determining the speed of light "in one direction".

\section{Stellar Aberration}

The stellar aberration $\alpha_{a}$ of any source for an observer at rest $v_{p i}=0$ in the reference frame concomitant to $\mathrm{CMB}$, is absent, since there is no observer's velocity perpendicular to the light flux of any observable source. At the same time, the stellar aberration $\alpha_{a p i}$ of any source for an observer moving at a pecular velocity $v_{p i}>0$ is determined by the perpendicular $\lrcorner v_{p i}$ to the direction to the source component of the observer's pecular velocity $v_{p i}$.

$$
\alpha_{p i}=\frac{\downarrow v_{p i}}{c}=\frac{v_{p i}}{c} \sin \psi
$$

where $\lrcorner v_{p i}-$ perpendicular to the direction to the source component of the observer's pecular $v_{p i}$ velocity;

$\psi$ - angle between the direction to the source and the observer's pecular velocity.

In this case, the total stellar aberration of the ground observer is determined, but not its components (diurnal, annual). It is excluded the century aberration estimated from the Galactic velocity of the solar system, since the Galactic velocity of the solar system is not equal to its pecular velocity.

\section{E. Doppler Effect}

The Doppler effect of any source for an observer at rest $v_{p i}=0$ in the reference frame concomitant to CMB depends only on the radial component of the velocity $v_{p r}$ of the observed object.

$$
z_{0}= \pm \frac{\Delta \lambda}{\lambda}= \pm \frac{v_{p r}}{c}
$$

where $z_{0}$ is the red/blue shift of the spectral line $\Delta \lambda$.

To determine the parameters of time and stellar aberration in any reference frame having a pecular velocity, it is important to know the value of its pecular velocity. Let us consider the possibilities of measuring the pecular velocity of the observer's reference frame.

\section{Measurement of the ObSERVER's PeCUlar VELOCITY}

Measurements of the Earth's peculiar velocity along CMB were first made after the discovery of CMB angular anisotropy according to its maximum in the RELIKT-1 experiment. The CMB dipole anisotropy is caused by the Doppler shift due to the Sun's motion relative to CMB. The accuracy achieved in measuring the Earth's peculiar velocity using the Doppler effect is such that the velocity of the Earth's rotation around the Sun stands out in its magnitude. The velocity of the observer in addition to the Doppler effect manifests itself in stellar aberration.

Let us compare in detail the Doppler effect and stellar aberration.

The Doppler effect (3) depends on the velocity of the observer relative to the light flux of the source in the direction of its propagation, including the Hubble velocity, so when the velocities of the observer and the source are equal, it is absent That is, in a moving inertial system with a source and an observer, the Doppler effect is not observable. It is observed in the presence of the relative velocity of the source and the observer along the direction of the light flux of the source.

The aberration (2) arises when the observer's velocity is only perpendicular to the direction of the light flux and does not depend on the velocity of the source. Knowing the magnitude of the stellar aberration it is possible to determine the velocity of the observer perpendicular to the direction of the light flux and vice versa.

That is, stellar aberration also occurs in a moving inertial system with a stationary source because it does not depend on the motion of the light source arriving at the telescope input at the time of measurement (Example of a star having a speed equal to that of the observer at the time of emission) [23], [24]. However, stellar aberration is observed only for distant sources [9]. And its measurement is made at transition of the observer from one reference frame to another only parts of its components (diurnal, annual), defined by difference of velocities of reference systems [24]. These velocities, and the aberrations corresponding to them, are components of the total, pecular velocity $v_{p}$ of the observer and the corresponding total aberration. Since it is impossible to measure the entire pecular velocity, the total aberration $\alpha=\arcsin \frac{v_{p}}{c}$ is considered non-observable.

To determine the possibility of determining the aberration without moving the observer from one reference frame to another by its change when the telescope is blocked by water, the experiment by Erie in 1871 was carried out. Its results confirmed the invariability of the stellar aberration angle predicted by Fresnel on the basis of his theory of partial entrainment of light by the medium. The analysis of such filling is carried out in detail, for example, in [25]. And filling the telescope not with isotropic matter, as in the Eri experiment, but with anisotropic uniaxial matter will allow one to observe stellar aberration without the observer's transition from one reference frame to another [26].

Due to the dependence of the refractive index of an anisotropic medium on the direction of propagation of the light flux, there is a change in this direction in the transverse motion of the anisotropic medium. The magnitude of the 
change depends on the direction and velocity of the medium.

The different ratio of the transverse entrainment velocity of the unusual light flux and its longitudinal velocity in a moving anisotropic medium, compared with their ratio for the ordinary light flux, causes a difference of the unusual beam aberration angle $\alpha_{e}$ from the ordinary beam aberration angle $\alpha_{o}$. Stellar aberration $\alpha_{e}$ along an unusual beam is different from stellar aberration $\alpha_{o}$ along an ordinary beam, as shown above:

$$
\alpha_{e}=\frac{n_{o}^{2}}{n_{e}^{2}} \alpha_{o}
$$

At known refractive indices $n_{o}, n_{e}$ and on the difference of deflection angles $\gamma_{o}, \gamma_{e}$ of the ordinary and unusual beams, the stellar aberration $\alpha_{o}$ of the observed object is determined without transition of the observer from one reference system to another [26].

$$
\alpha_{0}=\frac{\left(\gamma_{e}-\gamma_{o}\right)}{\frac{n_{o}^{2}}{n_{e}^{2}}-1}
$$

In this way, the aberration of stars is determined without the transition of the observer from one reference system to another, but stellar aberration is not observable for groundbased sources [9], [23], [24], [27], [28]. All stars differ from ground-based sources in their remoteness, and this primarily affects the curvature of the wave front of the light flux entering the telescope.

An analysis of the effect of the curvature of the wave fronts of the source light flux on the observability of the aberration of its light points to their determining influence on the aberration [29]. Stellar aberration is observed for distant sources because their wave front is almost flat when observed [9]. Consequently, the aberration of light from a nearby source should be observed if a flat wave front of its light flux is provided.

Analysis of possible devices for peculiar velocity meters based on filling the observation device with uniaxial anisotropic substance and without filling (according to the scheme of the Young interferometer, with Michelson interferometers with division of the amplitude and wave front of the light flux) indicates a higher sensitivity of devices on the scheme of the Jung interferometer and Michelson interferometer with division of the wave front of the light flux [30], [31].

Measuring the aberration of light in the inertial system makes it possible to directly measure the pecular velocity of the observer in the reference frame concomitant to CMB. Knowledge of the observer's pecular velocity is important for increasing the accuracy of determining the Hubble velocity, especially for objects located at relatively small distances

Using the Doppler effect, the total velocity of the observer, which consists of the Hubble velocity and the radial component of the observer's pecular velocity, is measured. Taking into account the radial component of the observer's pecular velocity increases the accuracy of the Hubble velocity.

Thus, if the Doppler effect is used to measure the total velocity of the observer-the Hubble velocity and the radial component of the observer's pecular velocity, the measurement of light aberration in an inertial system makes it possible to directly measure the observer's pecular velocity.

\section{CONCLUSION}

1. The microwave background radiation (CMB) and peculiarities of its relations with space-time determine the separation of the reference frame concomitant to CMB from all other reference frames.

2. The reference frame concomitant to CMB is complete, with its own clock. At any point of it the time is observable and synchronous. This provides the possibility of synchronization of the clocks of any other reference frames.

3. All the reference frames concomitant to any Universe object are within the reference frame concomitant to CMB. This is the only, at a given stage of cognition, zero pecular velocity reference frame that encompasses all others.

4. Time in it is fastest relative to time in any other reference frame, observable and measurable in any of them.

5. In the reference frame concomitant to $\mathrm{CMB}$ the relative velocity components of the two reference frames can be determined.

6. In it is possible: staging of experiments on measurement of light velocity "one way"; alignment of the astronomical reference frames HCRF and ICRF; use in problems and devices of deep space research.

\section{ACKNOWLEDGMENT}

The author is grateful to Dr. Yury Lvovich Bolotin for helpful discussions and support of the work.

\section{REFERENCES}

[1] A. Einstein, The principle of relativity and its implications in modern physics. (Transl.: Einstein A. Collection of scientific works Moscow, vol. 1, pp. 138-164, 1965).

[2] A. Michelson, Research on optics. Moscow: URSS, 2004, p. 200, pp. (34-40, 125-134).

[3] A. Michelson, F. Pease F. Pearson Repetition of the Michelson-Morley experiment, JOSA, vol. 18, no 3, pp.181-182, 1929.

[4] Masanori Sato. Single photon Michelson-Morley experiment via de Broglie-Bohm picture: An interpretation based on the hypothesis of frame dragging (2008) ArXiv: 0801.3138 [physics. gen-ph].

[5] J.A.S. Lima, F.D. Sasse, "Can Lorentz transformations be determined by the null Michelson-Morley result?", 2017, Arxiv.org/abs/1709.06864.

[6] G. A. Lorentz, Proc. Acad. Sci. Amsterdam, 1904, 6, 809. Transl. Electromagnetic phenomena in a system moving at any speed less than the speed of light. Lorentz G.A. Collection "The Principle of Relativity", GTTI, 1934.

[7] H. Poincar'e, La Science et l'Hypoth'ese. Flammarion, Paris, 1902. Poincaré A. About science. Moscow: Nauka publ., 1990, p.734.

[8] A. Einstein, "Principe de relativit'e et ses cons`equences dans la physique moderne," Arch. sci. phys. Natur., ser. 4, pp. 5-28, 125-144, 1910. (Transl.: Einstein A. Collection of scientific works Moscow, 1965. vol. 1, pp. 138-164).

[9] A. Einstein, "Zur Elektrodynamik der bewegten Korper," Annalen der Physik, vol. 17, pp. 891-921, 1905. (Transl.: Einstein A. Collection of scientific works. Moscow, 1965, vol. 1, pp. 7-35).

[10] A. Einstein, "Die Grundlage der allgemeinen Relanivitatstheorie, " Ann. Phys., vol. 49, pp. 769-822, 1916. (Transl.: Einstein A. Collection of scientific works. Moscow, 1965. vol. 1, pp. 452- 504). 
[11] A.D. Chernin, "How Gamov calculated the temperature of relict radiation, or a little about the art of theoretical physics," UFN, vol. 164, pp. 889-896, 1994.

[12] E. Khrutskaya, M. Khovrichev, I. Izmailov, A. Berezhnoi, "The Pulkovo program for the study of stars with large proper motions," GAO news, no. 219, Issue 4, pp. 355-360, 2009.

[13] D.P. Skulachev, "Correlation of the data on relic radiation anisotropy in the WMAP and Relict-1 experiments," UFN, vol. 180, pp. 389-392, 2010.

[14] M.R. Nolta et al., "Five-Year Wilkinson Microwave Anisotropy Probe (WMAP) Observations: Angular Power Spectra," ApJS, vol. 180, pp. 296-317, 2009

[15] Planck 2018 results. I. Overview and the Cosmological Legacy of Planck. Planck Collaboration, Astron. Astrophys. (2020), 641, A1.

[16] Planck 2018 results. Fossil radiation power spectra and probabilities. Planck Collaboration, Astron. Astrophys. (2020), 641, A6.

[17] A. Callinor, "Anisotropies in the Cosmic Microwave Backgrond," arXiv: astro-ph /0403344,33p. of Physics, no. 3, vol. 4, pp. 71-77, 2017.

[18] V.A. Rubakov, A. D. Vlasov, "What do learn from the CMB observations?" arXiv: astro-ph/ 1008.1704, p. 27.

[19] J. B. Zeldovich "Hot" model of the universe," UFN, vol. 89, p. 647$668,1966$.

[20] J.B. Zeldovich, I.D. Novikov, Structure and Evolution of the Universe. Moscow, 1975, p.736.

[21] A.D. Linde, "The Bloating Universe," UFN, vol. 144, Issue 2A, pp. 177-214, 1984

[22] V.G. Baryakhtar, Yu.L. Bolotin, A.V. Tur, V.V. Yanovsky. The Physical Fabric of the Universe. Kharkov: ISMA publ., 2010, p.512.

[23] W.I. Frankfurt, A.M. Frank, Optics of Moving Bodies. - Moscow: Nauka publ., pp. 76-112, 1972, (in Russian).

[24] B.N. Gimmelfarb, "To an Explanation of Stellar Aberration in the Theory of Relativity," Uspechi Physicheskich Nauk, vol. 51, 1953.

[25] T.S. Landsberg, General Physics. Optic. - Moscow: Nauka publ., 1976, p.928 (in Russian).

[26] V.M. Svishsh, "Licht aberration in optical anisotropic single-axis (uniaxial) medium," East European Journal, vol. 4, no. 3, 2017.

[27] A. Sommerfield, Vorlesungenuber Teoretische Physik. Rand 4. Optic von Arnold Sommerfeld. - Wisbaden, 1950, pp. 102-114.

[28] S. A. Tolchelnikova-Murri, "Stellar aberration and the observability of motions that cause it," Geodesy and Cartography, 1997, no. 7.

[29] V. Svishch, "Aberration of Light from a Terrestrial Source," Optics, vol. 7, no. 2, pp. 74-79, 2018. doi: 10.11648/j.optics.20180702.13.

[30] V.M. Svishch, "Speed Measurement in an Accompanying Reference system," East European Journal of Physics, no.2, pp. 81-88, 2020.

[31] V.M. Svishch, "About the Informative Parameters of Michelson Interferometers with the Division of Amplitude and the Wave Front," East European Journal of Physics, no. 3, vol. 5, pp. 24-31, 2018. 